\title{
Interstrain competition amongst mouse spermatozoa inseminated in various proportions, as affected by the genotype of the $Y$ chromosome
}

\author{
H. Krzanowska \\ Department of Genetics and Evolution, Institute of Zoology, Jagiellonian University, Karasia 6, \\ 30-060 Kraków, Poland
}

\begin{abstract}
Summary. Nonagouti $(\mathrm{KP} \times \mathrm{C} 57 \mathrm{BL}) \mathrm{F}_{1}$ hybrid females were artificially inseminated with a mixture of spermatozoa from males of the KE (nonagouti) and CBA (agouti) strains and the genotype of young was estimated by fur pigmentation. When KE and CBA spermatozoa mixed in the ratios of $1: 1,2: 1$ and $4: 1$ were inseminated after ovulation, $87 \%, 56 \%$ and $29 \%$ of progeny, respectively, were sired by CBA males, i.e. proportions of CBA progeny were significantly higher than ratios of CBA spermatozoa in the mixture. The surplus of CBA progeny was significantly less in females inseminated before ovulation, which may suggest that more rapid capacitation of CBA spermatozoa is partly responsible for their competitive advantage. In preparations from oviducal flushings of females killed $2-3 \mathrm{~h}$ after insemination, CBA spermatozoa (recognized by their shape) were found in similar proportions as in the inseminated mixture. There was therefore no evidence of their preferential selection at the uterotubal junction.

No competitive advantage of CBA spermatozoa occurred when they were inseminated with spermatozoa from males of the KE.CBA strain, congenic with KE but with the $\mathrm{Y}$ chromosome derived from the CBA strain. This indicates that genetic factors linked with the $\mathrm{Y}$ chromosome may influence competitive ability of spermatozoa.
\end{abstract}

\section{Introduction}

Spermatozoa of males from the inbred mouse strains, KE and CBA, are distinguishable under the microscope by their characteristic shapes, and differ significantly in the percentage of abnormal heads and in efficiency of fertilization (Krzanowska, 1969, 1970, 1981). While it has been reported that interstrain differences in morphology of normal spermatozoa are due to genes expressed through the germ line (Burgoyne, 1975), it is not known whether the same applies to differences in fertilizing ability of spermatozoa. To investigate this problem CBA $\leftrightarrow \mathrm{KE}$ aggregation chimaeras could be studied. If the genotype of germ cells is the main factor determining their quality, one could expect that the competition between KE and CBA spermatozoa in the ejaculate of chimaeric males would be reflected by the mixtures of spermatozoa from the males of these two strains. Therefore the aim of this preliminary study was to estimate relative fertilizing efficiency of $\mathrm{KE}$ and CBA spermatozoa when inseminated in different ratios.

Spermatozoa of the KE.CBA strain (congenic with KE but with the $\mathrm{Y}$ chromosome derived from the CBA strain) were also used to find out whether genetic factors linked with the $Y$ chromosome exert any influence on competitive ability of spermatozoa.

\section{Materials and Methods}

Adult, oestrous $(\mathrm{KP} \times \mathrm{C} 57 \mathrm{BL} / \mathrm{Kw}) \mathrm{F}_{1}$ hybrid females (genotype: $C \mathrm{Ca} a \mathrm{Bb}$ ) were artificially inseminated with the mixture of spermatozoa from 3-5-month-old males of two strains: CBA/Kw 
(subsequently referred to as CBA; genotype: $C C A A B B$ ) and $\mathrm{KE}(c c a a b b)$, or CBA and KE.CBA (26th backcross generation after introducing the CBA-derived $\mathrm{Y}$ chromosome onto the genetic background of the KE strain; genotype: $c c a a b b$ ). After insemination females were mated with vasectomized $(\mathrm{KP} \times \mathrm{C} 57 \mathrm{BL} / \mathrm{Kw}) \mathrm{F}_{1}$ hybrid males of proven sterility and inspected for the presence of a vaginal plug. Litter size was estimated at birth and 7 days later, when young were scored for fur pigmentation. Expression of agouti $(A)$ or nonagouti $(a a)$ alleles indicated paternity of CBA or KE (and KE.CBA) males, respectively.

Procedure. After killing the male by cervical dislocation, each cauda epididymidis was gently squeezed with forceps and then the contents of the vasa deferentia were expressed into $0.3 \mathrm{ml}$ boiled cows' milk. A small sample diluted with $3 \%(\mathrm{w} / \mathrm{v}) \mathrm{NaCl}$ was used to count the number of spermatozoa in a haemocytometer. Sperm suspensions from two males were mixed in the desired numerical ratios and insemination $(0.2 \mathrm{ml}$ per female) was performed by the method of Dziuk \& Runner (1960). In the main experiment females were maintained in a room with a dark period extending from $23: 00$ to $07: 00 \mathrm{~h}$ and they were inseminated at 11:00 h, i.e. after ovulation. In one experiment females, kept for 3 weeks in a room with a dark period from 14:00 to 02:00 $\mathrm{h}$, were inseminated at 19:00 h, i.e. before ovulation (no oviducal ova were found in 6 females killed at the time of insemination).

To estimate the efficiency of fertilization in natural matings, $F_{1}$ hybrid females $(\mathrm{KP} \times \mathrm{C} 57 \mathrm{BL} / \mathrm{Kw})$ were individually caged overnight with KE, KE.CBA or CBA males and killed 1 day after copulation. Ova flushed from the oviducts were examined. One-cell ova, if present, were fixed in acetic alcohol and stained with toluidine blue (Krzanowska, 1982); those in metaphase II of meiosis were counted as unfertilized.

Analysis of spermatozoa. A sample of each sperm mixture was diluted with $0.9 \%(\mathrm{w} / \mathrm{v}) \mathrm{NaCl}$, centrifuged, smeared on the slide, dried in air, fixed in acetic alcohol ( 3 parts absolute ethanol, 1 part glacial acetic acid) and stained with Feulgen reagent. The proportion of sperm heads from each strain, as recognized by their shapes (Fig. 1) was estimated from 400 spermatozoa counted per preparation. The percentages of abnormal sperm heads were counted on smears prepared from original samples before mixing.

Some females were killed $2-3 \mathrm{~h}$ after insemination. Contents of the torn ampullae and of the oviducts (flushed from the uterine end cut above the uterotubal junction) were placed on slides, dried in air and preparations were made as described above. All spermatozoa seen on these preparations were examined and classified, when possible, as being from males of the KE or CBA strain.

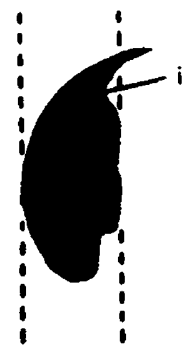

(a)

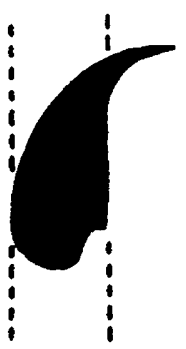

(b)

Fig. 1. Heads of spermatozoa from males of the CBA strain (a), and the KE and KE.CBA strains (b). The broken lines paralleling the ventral contour of the head help to visualize the difference in the curvature of the dorsal contour. CBA spermatozoa are also recognized by an invagination (i) in the apical part of the head (drawn from photographs of Feulgen-stained preparations). 


\section{Results}

\section{Analysis of litters after artificial insemination}

Of 77 inseminations followed by successful mating with a vasectomized male, $66 \%$ resulted in parturition and so sperm mixtures from $\geqslant 6$ pairs of males per group could be tested. Numbers of young were practically the same at birth as 7 days later when scored for pigmentation ( 1 young was missing in only 2 of 51 litters). Differences in litter size between groups were not significant (Table 1). When KE and CBA spermatozoa mixed in the ratios of $1: 1,2: 1$ and $4: 1$ were inseminated after ovulation, the percentages of young sired by CBA males were significantly higher than expected from percentages of CBA spermatozoa in the mixture. In the group inseminated before ovulation with a 1:1 sperm ratio, the proportion of young sired by CBA males was also higher than expected but significantly lower than in a corresponding group inseminated after ovulation (test of independence, $\chi^{2}=9.46, P<0.01$ ). CBA spermatozoa were therefore much more efficient in competition with $\mathrm{KE}$ spermatozoa, especially when inseminated after ovulation.

Table 1. Proportions of young sired by CBA males after artificial insemination with a mixture of spermatozoa from two strains

\begin{tabular}{|c|c|c|c|c|c|c|c|c|}
\hline \multicolumn{2}{|c|}{ Sperm mixture } & \multirow{2}{*}{$\begin{array}{c}\text { Mean } \pm \text { s.d. } \\
\text { no. of } \\
\text { spermatozoa } \\
\left(\times 10^{-6}\right) \\
\text { inseminated }\end{array}$} & \multirow{2}{*}{$\begin{array}{l}\text { No. of } \\
\text { litters }\end{array}$} & \multirow{2}{*}{$\begin{array}{l}\text { Mean } \pm \text { s.d. } \\
\text { litter size }\end{array}$} & \multicolumn{2}{|c|}{$\begin{array}{l}\text { No. of young } \\
\text { of genotypes }\end{array}$} & \multicolumn{2}{|c|}{$\begin{array}{c}\% \text { of young sired by } \\
\text { CBA males }\end{array}$} \\
\hline Source & Ratio & & & & $a a$ & $A a$ & Expected + & Obtained \\
\hline $\mathrm{KE}: \mathrm{CBA}$ & $1: 1 \dagger$ & $3.5 \pm 0.96$ & 9 & $7 \cdot 3 \pm 3 \cdot 1$ & 24 & 42 & 50 & $63 \cdot 4^{*}$ \\
\hline $\mathrm{KE}: \mathrm{CBA}$ & $\begin{array}{l}1: 1 \\
2: 1 \\
4: 1\end{array}$ & $\begin{array}{l}3.7 \pm 1.47 \\
3.6 \pm 1.65 \\
4 \cdot 1 \pm 1.68\end{array}$ & $\begin{array}{r}9 \\
8 \\
10\end{array}$ & $\begin{array}{l}8 \cdot 5 \pm 2 \cdot 3 \\
8 \cdot 0 \pm 2 \cdot 8 \\
7 \cdot 5 \pm 2 \cdot 1\end{array}$ & $\begin{array}{l}10 \\
28 \\
53\end{array}$ & $\begin{array}{l}67 \\
36 \\
22\end{array}$ & $\begin{array}{l}50 \\
33 \\
20\end{array}$ & $\begin{array}{l}87 \cdot 0^{* * *} \\
56 \cdot 2^{* *} \\
29 \cdot 3^{*}\end{array}$ \\
\hline KE.CBA:CBA & $\begin{array}{l}1: 1 \\
2: 1\end{array}$ & $\begin{array}{l}3 \cdot 6 \pm 1 \cdot 37 \\
4 \cdot 5 \pm 2 \cdot 80\end{array}$ & $\begin{array}{l}8 \\
7\end{array}$ & $\begin{array}{l}7 \cdot 6 \pm 2 \cdot 9 \\
8 \cdot 6 \pm 2 \cdot 4\end{array}$ & $\begin{array}{l}30 \\
44\end{array}$ & $\begin{array}{l}31 \\
16\end{array}$ & $\begin{array}{l}50 \\
33\end{array}$ & $\begin{array}{l}50 \cdot 8 \\
26 \cdot 7\end{array}$ \\
\hline
\end{tabular}

+ Insemination performed before ovulation; in all other groups insemination was after ovulation.

$¥$ Assuming that each spermatozoon in the mixture had an equal chance of fertilizing an egg.

${ }^{*} P<0.05,{ }^{* *} P<0.01$ compared with value expected $\left(\chi^{2}\right.$ test $)$.

In contrast, CBA spermatozoa showed no advantage in competition with KE.CBA spermatozoa (Table 1). Inseminations with a 1:1 sperm ratio resulted in equal proportions of young sired by the males of each strain and for the ratio of KE.CBA:CBA $=2: 1$, the proportion of young sired by CBA males was even lower than expected (difference not significant).

It was tested whether the proportion of offspring sired by competing males could be predicted for the given sperm ratio by the formula established with birds. When inseminating hens with sperm mixtures from males of two breeds, C and L, Martin, Reimers, Lodge \& Dziuk (1974) came to the conclusion that ratios of spermatozoa and proportions of offspring are mathematically related according to the following equation:

$\% \mathrm{C}$ offspring calculated $=$

100 (\% $\%$ offspring from $1 \mathrm{C}: 1 \mathrm{~L}$ sperm ratio) $(\% \mathrm{C}$ sperm in calculated ratio)/(\% $\mathrm{C}$ offspring from $1 \mathrm{C}: 1 \mathrm{~L}$ sperm ratio) $(\% \mathrm{C}$ sperm in calculated ratio) $+(\% \mathrm{~L}$ offspring from $1 \mathrm{C}: 1 \mathrm{~L}$ sperm ratio) $(\% \mathrm{~L}$ sperm in calculated ratio)

As shown in Fig. 2 this simple equation could not be applied to the present results with mice. With the diminishing ratio of CBA spermatozoa, their chances to fertilize ova dropped much more rapidly than predicted from inseminations with a 1:1 ratio, the slope being slightly steeper for the $\mathrm{KE}$ and CBA $(b=1.93)$ than for KE.CBA and CBA $(b=1.41)$ sperm mixtures. 


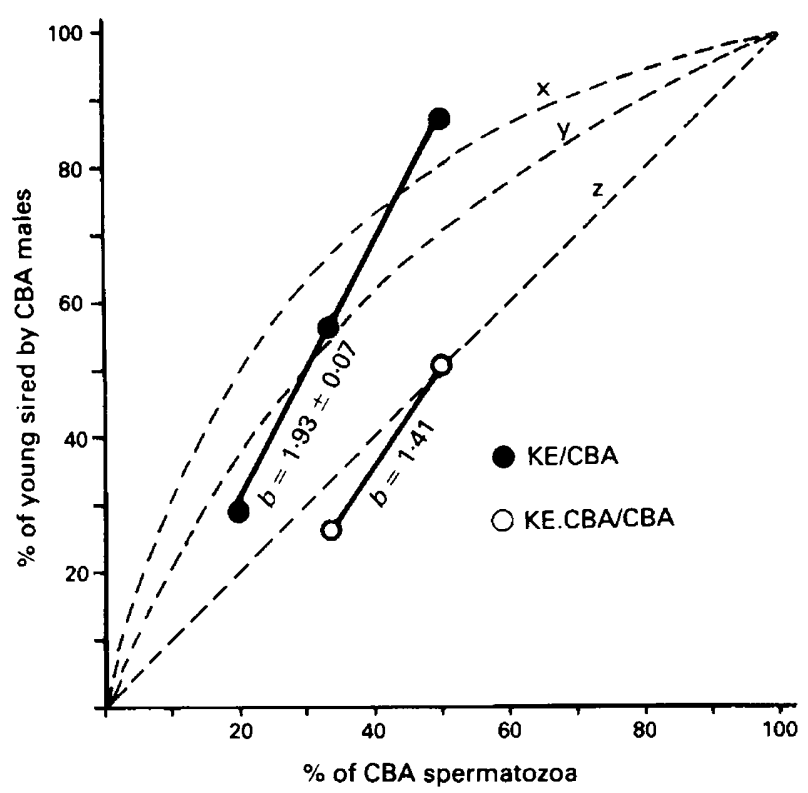

Fig. 2. Relationship between the percentage of CBA spermatozoa in a sperm mixture inseminated after ovulation and the percentage of young sired by CBA males. $x, y, z$, Curves predicted by the equation of Martin et al. (1974) on the assumption that insemination with a 1:1 sperm ratio gives $80 \%, 70 \%$ and $50 \%$ of CBA progeny, respectively.

\section{Analysis of spermatozoa}

Percentages of abnormal sperm heads in original samples (before mixing) were typical of a given strain and amounted to $19 \%$ for $\mathrm{KE}, 9 \%$ for KE.CBA and $5 \%$ for CBA. In preparations from sperm mixtures. CBA spermatozoa were distinguished from KE as well as from KE.CBA spermatozoa by head shape (Fig. 1). Severely misshapen heads (amounting to $1-3 \%$ in $\mathrm{KE}$ and $1-2 \%$ in CBA) were not counted. For sperm samples mixed in the ratios (KE:CBA or KE.CBA:CBA) of $1: 1,2: 1$ and $4: 1$, the following percentages of CBA spermatozoa were scored on preparations (Mean \pm s.d.): $48 \pm 3 \cdot 5,31 \pm 3 \cdot 6$ and $20 \pm 2 \cdot 2$, respectively. For the $1: 1$ and $2: 1$ sperm ratios, therefore, CBA spermatozoa appeared in slightly lower proportions than those calculated for the insemination mixtures. It seems unlikely that KE spermatozoa were classified wrongly as CBA spermatozoa, but some slightly abnormal CBA sperm heads could have been counted as KE which would explain underestimation of the percentage of CBA spermatozoa in the preparations.

In 6 females inseminated after ovulation with a 1:1 sperm ratio and killed $2-3 \mathrm{~h}$ after insemination, motile spermatozoa were seen in the uterus. In preparations from the oviducal flushings only normal sperm heads were seen, all of them were classified and the following proportions of CBA spermatozoa were scored: $62 / 118,28 / 76,67 / 145$ for KE and CBA sperm mixtures, and 46/94, 34/76, 54/90 for KE.CBA and CBA mixtures. There was therefore no evidence of more efficient transport of CBA spermatozoa to the oviduct.

In preparations from the contents of the ampullae all ova were surrounded by dense cumuli. Spermatozoa (1-2) were seen inside 14 out of 62 cumuli and 6 ova were already penetrated. In these preparations it was difficult to identify the shapes of spermatozoa because their heads were usually distorted by the curvature of the ova. Although some were recognized as CBA spermatozoa, their proportions could not be reliably estimated. 
Table 2. Efficiency of fertilization in natural matings of $(\mathrm{KP} \times \mathrm{C} 57 \mathrm{BL} / \mathrm{Kw}) \mathrm{F}_{1}$ hybrid females with $\mathrm{KE}$, KE.CBA or CBA males

\begin{tabular}{lccccc}
\hline & $\begin{array}{c}\text { No. of } \\
\text { pairs }\end{array}$ & Mean \pm s.d. no. of & & \multicolumn{2}{c}{$\begin{array}{c}\text { No. of supplementary } \\
\text { spermatozoa:no. of ova* }\end{array}$} \\
of male & mated & ova per female & $\begin{array}{c}\text { Proportion (\%) of } \\
\text { fertilized ova* }\end{array}$ & Fertilized & Unfertilized \\
\hline KE & 11 & $9 \cdot 4 \pm 1 \cdot 44$ & $84: 96(87 \cdot 5)$ & $38: 84$ & $11: 12$ \\
KE.CBA & 12 & $9 \cdot 2 \pm 1 \cdot 80$ & $89: 93(95 \cdot 7)$ & $45: 89$ & $2: 4$ \\
CBA & 11 & $9 \cdot 1 \pm 2 \cdot 67$ & $90: 90(100 \cdot 0)$ & $16: 90$ & - \\
\hline
\end{tabular}

* Sterile copulations (1-2 per group) not included.

\section{Efficiency of fertilization in natural matings}

Efficiency of fertilization of KE males was significantly lower than that of CBA males which fertilized $100 \%$ of ova (Table 2). The performance of KE.CBA males was intermediate and not significantly different from those of KE or CBA males. After matings with KE or KE.CBA males about $30 \%$ of eggs (fertilized and unfertilized) contained 1-3 supplementary spermatozoa in the perivitelline space. Supplementary spermatozoa (no more than 1 per egg) were rarely seen after matings with CBA males.

\section{Discussion}

Heterospermic insemination with a mixture of spermatozoa from different males is a sensitive method of evaluating their relative fertilizing efficiency; differences between males in their heterospermic performance remain relatively constant over periods of time and are usually positively correlated with homospermic fertility of respective males (reviewed by Beatty, 1970). The present results show that CBA spermatozoa were much more efficient in competition with KE spermatozoa. This could be expected from results of natural mating because CBA males regularly give $100 \%$ fertilization, while KE males fertilize 76-100\% of ova, depending on the strain of female (Krzanowska, 1970). KE spermatozoa were also significantly less efficient than spermatozoa of inbred KP and outbred males in heterospermic inseminations (Musialek, 1969).

Vigorous $(\mathrm{KP} \times \mathrm{C} 57 \mathrm{BL} / \mathrm{Kw}) \mathrm{F}_{1}$ hybrid females, not directly related to $\mathrm{KE}$ or $\mathrm{CBA}$ strains, were chosen for insemination because of their good maternal performance and nonagouti genotype. They appeared to be suitable for studying competition between KE and CBA spermatozoa in the mixtures, although these females gave only slightly lower percentages of fertilization in natural matings with KE males than with CBA males (Table 2).

As discussed by Buehr \& McLaren (1984) there are a number of possible ways in which one sperm type might prove more successful than another in producing young, e.g. more efficient sperm transport in the female tract, more rapid sperm capacitation or differential survival of embryos. In the present experiments there was no evidence of preferential selection of CBA spermatozoa in their transport to the oviduct. On the contrary, KE spermatozoa seem to be more efficient: when inseminated they contained a high proportion of abnormal forms, the majority of which are prevented from passing the uterotubal junction (Krzanowska, 1974) and yet normal KE spermatozoa appeared in the oviducts in proportions similar to those of CBA spermatozoa (for a $1: 1$ sperm ratio). In females killed $2-3 \mathrm{~h}$ after insemination penetration of ova was just beginning and thus fertilizing spermatozoa had to be recruited from those found in the oviduct at that time. Because it was not possible to calculate the proportions of CBA and KE spermatozoa in proximity to the eggs, it cannot be excluded that a higher efficiency in traversing the cumulus could be responsible for the higher fertilization rate of CBA spermatozoa. 
On the other hand, the results of inseminations performed before and after ovulation seem to suggest that KE spermatozoa require a longer time for capacitation. Their competitive disadvantage was significantly reduced in the group inseminated $1 \mathrm{~h}$ before the midpoint of the dark period, i.e. 4-7 $\mathrm{h}$ before the expected ovulation (calculated after Braden, 1957), when they had several hours left for successful capacitation. However, it is also possible that the capacitation rate of KE spermatozoa could be in some way affected by the postovulatory conditions of the oviduct. It is known that the time of insemination may influence relative fertilizing efficiency of spermatozoa of different genotypes (e.g. Braden, 1958; Buehr \& McLaren, 1984).

When looking for the possible mechanisms underlying differential fertilizing ability of $\mathrm{KE}$ and CBA spermatozoa, one should also consider the difference in the number of supplementary spermatozoa in the perivitelline space. As shown earlier (Krzanowska, 1970) and in Table 2, after normal matings with CBA males supplementary spermatozoa occur only rarely which means that the first spermatozoon entering the perivitelline space is likely to be the fertilizing one. In contrast, after matings with KE males supplementary spermatozoa were often seen both in fertilized and unfertilized ova which suggests that failure to penetrate the vitellus might contribute to competitive disadvantage of KE spermatozoa. However, the strength of this argument is weakened by the fact that many supplementary spermatozoa were also seen after matings with KE.CBA males and yet their spermatozoa competed successfully with those of CBA males.

Differential survival of embryos is not likely to account for the surplus of progeny sired by CBA males because the difference between the number of ova ovulated ( $\sim 9$ per female, Table 2$)$ and the number of young born $(\sim 8$ per female, Table 1$)$ was slight.

The KE.CBA strain, congenic with $\mathrm{KE}$ but with the $\mathrm{Y}$ chromosome introduced from CBA, was originally developed to test the role of the $\mathrm{Y}$ chromosome in determining the level of sperm abnormalities. KE.CBA strain males are characterized by a lower percentage of abnormal sperm heads than are KE males and are more efficient at fertilization in natural matings, although their performance is slightly poorer than that of CBA males (Krzanowska, 1969). The present results show that the introduction of the $\mathrm{Y}$ chromosome from the CBA to the KE strain resulted in an improved competitive ability of the spermatozoa in this strain, so that they had equal chances of fertilization as CBA spermatozoa when mixed in a 1:1 ratio. This suggests that genetic factors linked with the $\mathrm{Y}$ chromosome may influence functional competence of spermatozoa.

This work was supported by the Polish Academy of Sciences within the project MR II-9.

\section{References}

Beatty, R.A. (1970) The genetics of the mammalian gamete. Biol. Rev. 45, 73-119.

Braden, A.W.H. (1957) The relationship between the diurnal light cycle and the time of ovulation in mice. J. exp. Biol. 34, 177-188.

Braden, A.W.H. (1958) Influence of the time of mating on the segregation of alleles at the $\mathrm{T}$ locus in the house mouse. Nature, Lond. 181, 786-787.

Buehr, M. \& McLaren, A. (1984) Interlitter variation in progeny of chimaeric male mice. J. Reprod. Fert. 72, 213-221.

Burgoyne, P.S. (1975) Sperm phenotype and its relationship to somatic and germ line genotype: a study using mouse aggregation chimaeras. Devl Biol. 44, 63-76.

Dziuk, P.J. \& Runner, M.N. (1960) Recovery of blastocysts and induction of implantation following artificial insemination of immature mice. $J$. Reprod. Fert. 1, 321-331.

Krzanowska, H. (1969) Factor responsible for spermatozoan abnormality located on the $Y$ chromosome in mice. Genet. Res. 13, 17-24.
Krzanowska, H. (1970) Relation between fertilization rate and penetration of eggs by supplementary spermatozoa in different mouse strains and crosses. $J$. Reprod. Fert. 22, 199-204.

Krzanowska, H. (1974) The passage of abnormal spermatozoa through the uterotubal junction of the mouse. J. Reprod. Fert. 38, 81-90.

Krzanowska, H. (1981) Sperm head abnormalities in relation to the age and strain of mice. J. Reprod. Fert. 62, 385-392.

Krzanowska, H. (1982) Toluidine blue staining reveals changes in chromatin stabilization of mouse spermatozoa during epididymal maturation and penetration of ova. J. Reprod. Fert. 64, 97-101.

Martin, P.A., Reimers, T.J., Lodge, J.R. \& Dziuk, P.J. (1974) The effect of ratios and numbers of spermatozoa mixed from two males on proportions of offspring. J. Reprod. Fert. 39, 251-258.

Musialek, B. (1969) Reproductive competition among males of different strains in the mouse. Genet. Polon. 10, $180-183$ 